\title{
Constraining challenging regions of the SUSY parameter space with the CMS experiment
}

\section{Peter Eduard Meiring ${ }^{* \dagger}$}

University of Zurich,

Winterthurerstrasse 190, Zurich, Switzerland

E-mail: Peter.eduard.meiring@cern.ch

Supersymmetric models are characterized by a strong diversity of experimental signatures. Since general-purpose searches have not yet given any clear indication of new physics, dedicated methodologies and tools have been developed to target the regions of the parameter space where the analysis is most challenging and SUSY might still lie undetected. This contribution will describe relevant examples among searches performed by the CMS Collaboration using the full dataset of proton-proton collisions collected during the Run 2 of the LHC at a center-of-mass energy of 13 $\mathrm{TeV}$.

*** The European Physical Society Conference on High Energy Physics (EPS-HEP2021) ***

*** 26-30 July 2021 ***

*** Online conference, jointly organized by Universität Hamburg and the research center DESY ***

\footnotetext{
* Speaker

${ }^{\dagger}$ On behalf of the CMS Collaboration
} 


\section{Introduction}

Despite large amounts of collision data provided by the Large Hadron Collider (LHC) and numerous searches, no hint for New Physics (NP) at the electroweak scale has been observed yet. Instead, powerful constraints are placed on supersymmetric particle masses. The traditional analysis strategy that leads to such strong constraints is to search for NP in collision events with high visible energy as well as high invisible transverse momentum $p_{\mathrm{T}}^{\text {miss }}$. This strategy, however, does not allow the full coverage of the phase-space where supersymmetry (SUSY) still may reside. The very corners of the phase-space have to be explored, but targeting the corresponding SUSY realizations comes with ever-increasing experimental challenges. In the following, three NP searches will be highlighted, each tackling a challenge specific to the SUSY parameter space they target. All searches are performed with data collected with the CMS experiment [1] during LHC Run 2 (2016-2018).

\section{Compressed Higgsinos}

A natural SUSY realization would solve the hierarchy problem with a minimal amount of fine-tuning required. It suggests the presence of Higgsinos at the electroweak scale with small mass-splittings. Signatures of light, mass-compressed particles consist of only moderate amounts of $p_{\mathrm{T}}^{\text {miss }}$ in combination with low $p_{\mathrm{T}}$ visible decay products. Ref. [2] targets the topology of Figure 1 (left) with one opposite-sign same-flavor pair of leptons (e/ $\mu$ ) from the off-shell $\mathrm{Z}$ boson and a possible third lepton from the off-shell $\mathrm{W}$ boson. Challenges arise already at the online event selection due to the absence of high visible $p_{\mathrm{T}}$ or high $p_{\mathrm{T}}^{\text {miss }}$. To overcome this, firstly, a dedicated $\mu \mu+p_{\mathrm{T}}^{\text {miss }}$ cross-trigger has been designed to increase acceptance to low $p_{\mathrm{T}}^{\text {miss }}$ events. Furthermore, the requirement of a jet from initial state radiation (ISR) in back-to-back configuration with the sparticle pair, induces enough boost for pure $p_{\mathrm{T}}^{\text {miss }}$ triggers. This naturally decreases the event yields of a process that already has a low production cross-section to begin with. Large statistical uncertainties are therefore a reoccurring theme of this search.

The most challenging feature, however, comes from the use of low $p_{\mathrm{T}}$ leptons $\left(3.5<p_{\mathrm{T}}<\right.$ $30 \mathrm{GeV}$ ) in the final state. Many non-prompt lepton candidates - predominantly from heavy flavor decays - constitute the main background. To estimate this background, a tight-to-loose method is used; Firstly, a lepton misidentification probability (FR) is measured in a QCD-enriched
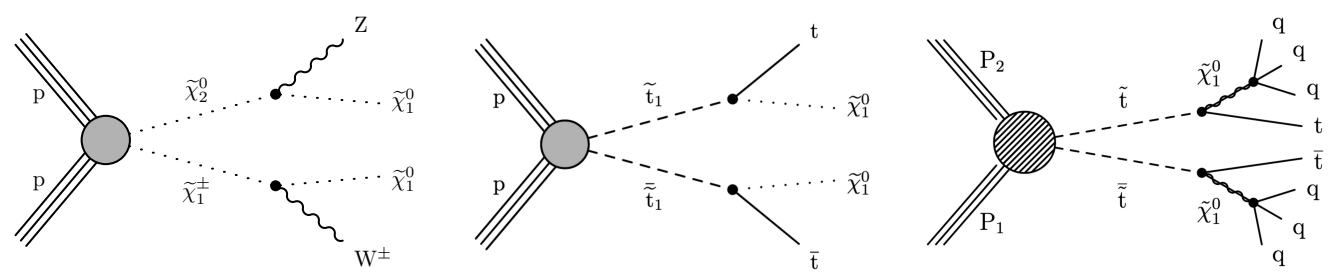

Figure 1: Signal topologies targeted by the searches highlighted in this contribution. Left: Pair-produced electroweakinos, targeted in the search for compressed Higgsinos [2]. Middle: Pair-produced stops decaying to a top-pair and stable neutralinos, targeted in the search for light stops in the Top Corridor [3]. Right: R-parity violating stops decaying to large numbers of jets, targeted in Ref. [4]. 
measurement region (MR). A signal-like application region (AR) is then defined by inverting the tight lepton identification (ID) requirement of the signal region (SR). Applying the FR on events in the AR then allows to estimate the background of non-prompt leptons in the signal region (SR). This method relies heavily on the definition of the loose- and tight lepton ID criteria. Their choice is very delicate when dealing with low $p_{\mathrm{T}}$ leptons. This search uses several handles to customize the ID definition; Firstly, an absolute isolation cut is applied i.c.w. a $p_{\mathrm{T}}$ dependent relative isolation cut to control the overwhelming background of non-isolated non-prompt leptons at the lowest $p_{\mathrm{T}}$ range. Furthermore, the 3D impact parameter (and significance) are used to further reduce the non-prompt lepton contamination. Lastly, $p_{\mathrm{T}}$-dependent working points of a dedicated multivariate electron ID allow for additional control of the tightness of the selected electrons. Special care must be taken when tuning each of these parameters in order to achieve closure of the method.

Since the tight-to-loose method can attribute a negative transfer factor to some events, statistical uncertainties are increased in the analysis regions that are already low in event yields. To mitigate this effect, modifications to the method are applied to exploit the large statistical power of Monte Carlo (MC) simulations; Instead of applying the transfer factor to data in the AR, it is applied to samples of non-prompt leptons from simulated events, which decreases the statistical uncertainties. The final background estimation is compared with data in Figure 2.

The signal extraction of this search is based on a likelihood fit binned in invariant dilepton mass $m_{l l}$ and $p_{\mathrm{T}}^{\text {miss }}$. Largely due to the dedicated non-prompt lepton estimation method, strong exclusion limits could be set at the lowest mass-splittings; In the Higgsino Simplified Model, next-to-lightest supersymmetric particle (NLSP) masses up to $215 \mathrm{GeV}$ at a mass-splitting of $5 \mathrm{GeV}$ and up to 150 $\mathrm{GeV}$ at a mass-splitting of $3 \mathrm{GeV}$ are excluded, as is shown in Figure 2 (right).
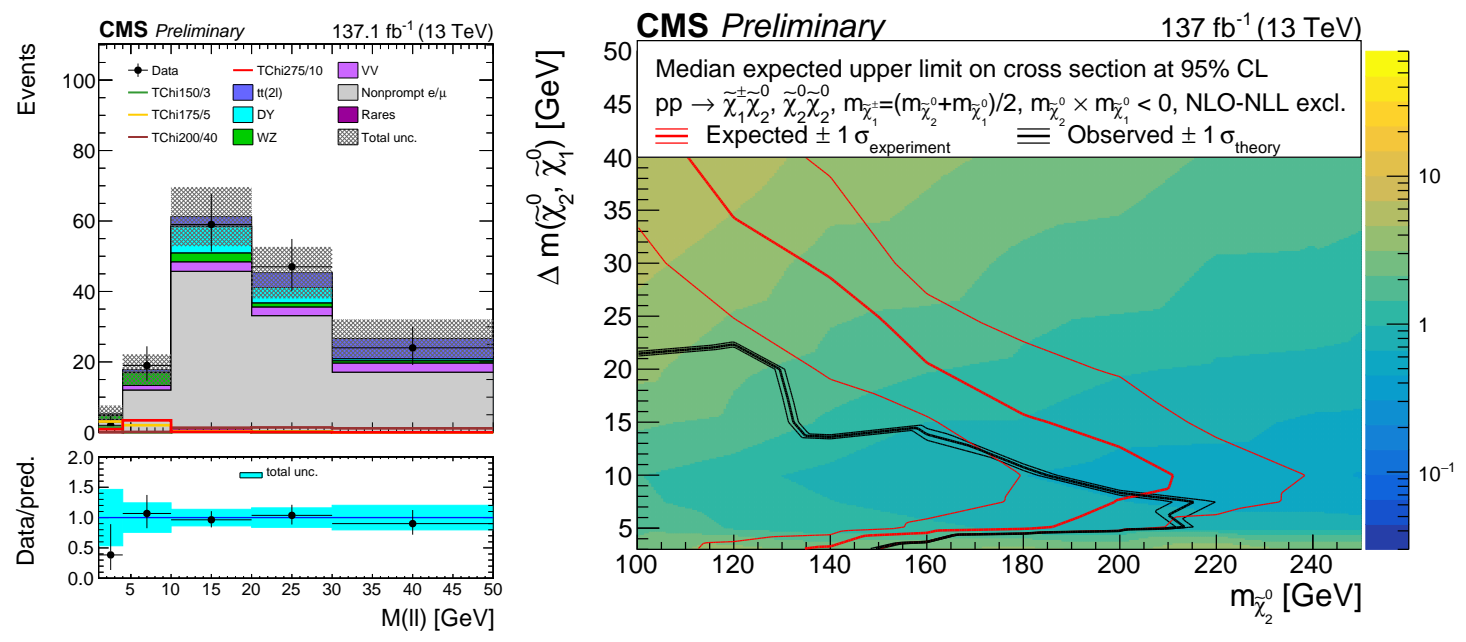

Figure 2: Left: A post-fit SR distribution of $m_{l l}$ is shown, corresponding to $200<p_{\mathrm{T}}^{\text {miss }}<240 \mathrm{GeV}$ and full Run 2 luminosity. Both statistical and experimental components are included in the uncertainties. Right: The exclusion contours at $95 \%$ confidence level (CL) on the $\widetilde{\chi}^{ \pm} \widetilde{\chi}_{2}^{0}$ production cross-section, corresponding to the Higgsino Simplified Model [2]. 


\section{Light Stops in Top Corridor}

Another challenging corner of the SUSY parameter space to be explored is the so-called "Top Corridor", referring the scenarios where $m_{\widetilde{t}}-m_{\widetilde{\chi}_{1}^{0}} \approx m_{t}$. Pair-produced stops in the Top Corridor could decay to a stable LSP neutralino, which in turn could serve as a dark matter candidate. This part of the parameter space is particularly challenging to target if the stops in addition are light; this results in a final state signature that strongly resembles Standard Model (SM) $t \bar{t}$ production in combination with low amounts of $p_{\mathrm{T}}^{\text {miss }}$. Distinguishing the signal from kinematically very similar background therefore constitutes the key challenge of the search described in Ref. [3].

To distinguish the signal from irreducible SM $t \bar{t}$, small kinematic differences are optimally exploited by means of a Deep Neural Network (DNN) with 7 hidden layers, using 11 kinematic variables as input. It should be noted, however, that the shapes of several input features, such as $p_{\mathrm{T}}^{\text {miss }}$ and $m_{\mathrm{T} 2}$, strongly depend on the choice of $m_{\widetilde{\chi}_{1}^{0}}$. Consequently, the discrimination power of those input variables varies as function of the mass hypotheses of signal in the Top Corridor. A single DNN trained for the full parameter space would therefore be sub-optimal. To optimize the DNN performance, both $m_{\widetilde{t}}$ and $m_{\widetilde{\chi}_{1}^{0}}$ and are included as additional input features to the training. The DNN is hence referred to as a parametric DNN [5]. For events from the $t \bar{t}$ background sample, these mass parameters are randomly assigned, based on the probability distribution of the signal sample. This strategy allows the parametric DNN to optimally benefit from the discrimination power at every mass hypothesis, resulting in a DNN score shape that differs for both signal and background as function of $m_{\widetilde{t}}$ and $m_{\widetilde{\chi}_{1}^{0}}$.

The output score provides maximum shape difference between signal and background and is therefore used as fit variable for the signal extraction, performed in 9 channels of data-taking period and final state lepton flavor compositions. Figure 3 (left) shows a post-fit SR distribution of the DNN score, corresponding to a single mass hypothesis. A good agreement between data and background estimations is observed. The final exclusion limits at $95 \%$ confidence level (CL) are shown in Figure 3 (right); light stops are fully excluded from the Top Corridor.
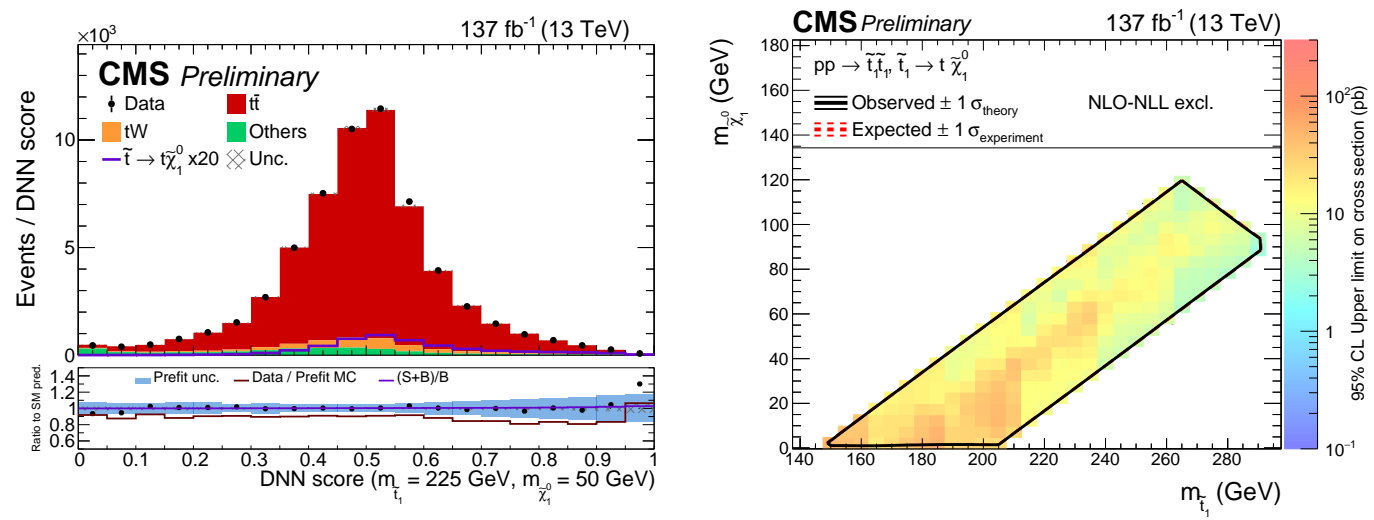

Figure 3: Left: Post-fit DNN score in the SR, corresponding to the mass hypothesis $m_{\widetilde{t}}, m_{\widetilde{\chi}_{1}^{0}}=225,50 \mathrm{GeV}$. Signal is scaled by a factor 20. Right: Exclusion limits at $95 \% \mathrm{CL}$ on the signal cross-section. Light stops in the Top Corridor are fully excluded [3]. 


\section{Stops in R-parity violating models}

The signal topology of pair produced sparticles that decay to a stable LSP is a direct consequence of the R-parity conservation. This property of the theory is assumed by both analyses described above. However, several SUSY theories predict R-parity violation (RPV), resulting in unstable LSPs decaying to SM particles, an example of which is shown in Figure 1 (right). The search of Ref. [4] targets exactly this process with final state signatures containing a $t \bar{t}$ pair in addition to large jet multiplicity. Since MC generators generally cannot compute matrix elements with high jet multiplicity, these events have to be simulated by means of hadronization, which results in a poor description of jet activity. Ref. [4] therefore estimates the main background (SM $t \bar{t}+$ jets) from data instead of relying on MC simulations. It should be noted however, that the large kinematic similarities between signal and background hinder the construction of a signal-free control region that could be used for the data-driven background estimation.

To overcome this challenge, the search utilizes a neural network (NN) to discriminate signal from $t \bar{t}+$ jets background. The signal region is then divided in bins of jet multiplicity $N_{j}$ as well as the NN output score $S_{\mathrm{NN}}$. By construction, bins with low $N_{j}$ and low $S_{\mathrm{NN}}$ are background-enriched, whereas bins with high $N_{j}$ and high $S_{\mathrm{NN}}$ are signal enriched. The low $S_{\mathrm{NN}}$ region can be used as quasi-CR to extract the shape of $N_{j}$, which is used during the simultaneous fit to data in the full SR. The key assumption that allows this analysis strategy is the independence between $N_{j}$ and $S_{\mathrm{NN}}$.
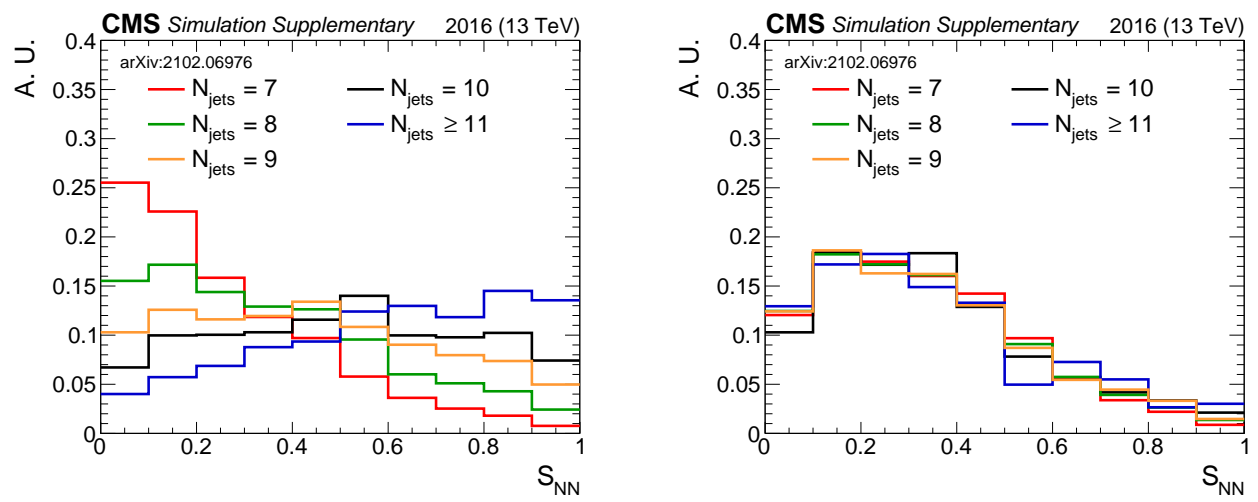

Figure 4: $S_{\mathrm{NN}}$ as function of $N_{j}$ for a NN trained without (left) and with (right) gradient reversal [4].

Figure 4 (left) shows $S_{\mathrm{NN}}$ as function of $N_{j}$ for a NN that was trained purely to classify events as signal or $t \bar{t}+$ jets background. The observed trend clearly breaks the key assumption described above. However, a technique referred to as gradient reversal [6] can be employed to establish independence of $S_{\mathrm{NN}}$ on $N_{j}$ when training the NN. Two NNs are combined: the first NN serves to classify events as signal or background as before, but a second $\mathrm{NN}$ is added that aims to predict $N_{j}$ in each event. The total loss-function therefore gains an additional term that effectively penalizes any predictive power in $N_{j}$. The effect of this technique can be seen in Figure 4 (right); Gradient reversal clearly cures the dependency, resulting in similar shapes of $N_{j}$ in each $S_{\mathrm{NN}}$ bin of the SR.

For the final fit to data, the fixed $N_{j}$ shape of the $t \bar{t}+$ jets background is extracted from the low $S_{\mathrm{NN}}$ regions. Other backgrounds, such as $\mathrm{tW}$ or diboson production, are estimated directly from MC and QCD multijet background is estimated with a dedicated CR. A simultaneous fit is 
performed and the post-fit background estimations show good agreement with the data, as is shown in Figure 5 (left). Results are interpreted in the benchmark RPV model and the exclusion limit is shown in Figure 5 (right). Stop masses up to $670 \mathrm{GeV}$ are excluded, which constitutes the most powerful limit to date.
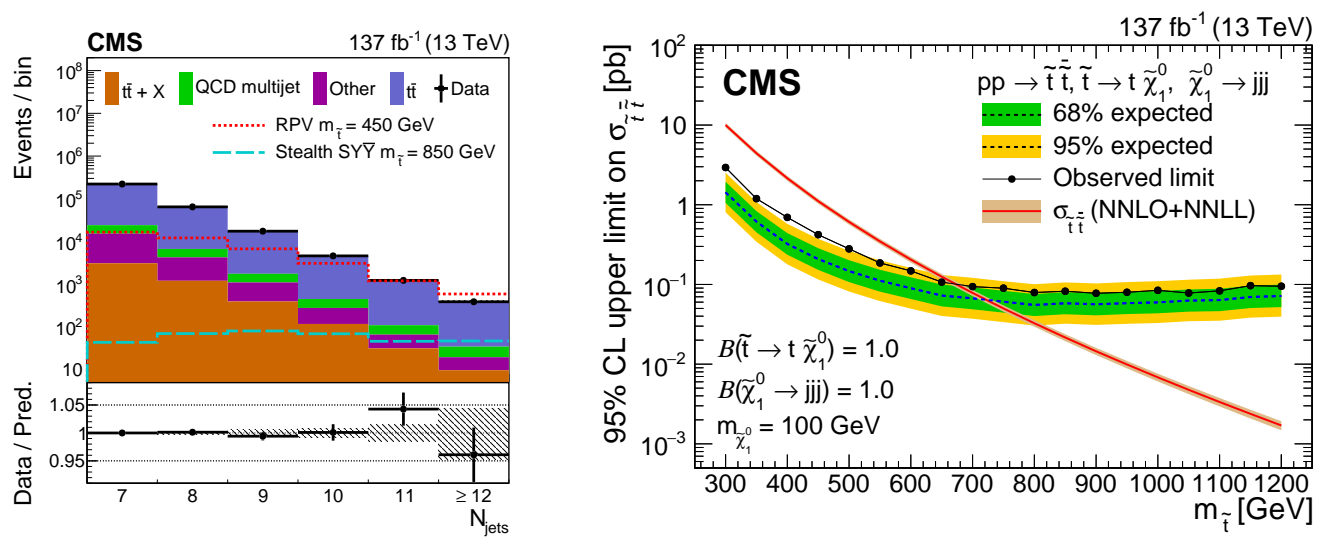

Figure 5: Left: Post-fit SR distribution of $N_{j}$, summed over all $S_{\mathrm{NN}}$ bins. Right: Upper limits at $95 \% \mathrm{CL}$ on the stop pair production cross-section. Stop masses up to $670 \mathrm{GeV}$ are excluded in the RPV model [4].

\section{Summary}

To explore the corners of the phase-space where SUSY may still reside, more unconventional signatures have to be targeted, which are accompanied by increasing experimental challenges. Three searches were presented that utilize improved background estimation techniques and innovative analysis strategies to overcome these challenges. All searches were based on the data collected by the CMS experiment during 2016-2018. No significant deviations from the expected backgrounds were observed and strong constraints were set on sparticle masses for the SUSY models considered.

\section{References}

[1] CMS collaboration, The CMS Experiment at the CERN LHC, JINST 3 (2008) S08004.

[2] CMS collaboration, Search for physics beyond the standard model in final states with two or three soft leptons and missing transverse momentum in proton-proton collisions at $13 \mathrm{TeV}$, https://cds.cern.ch/record/2758359.

[3] CMS collaboration, Combined searches for the production of supersymmetric top quark partners in proton-proton collisions at $\sqrt{s}=13 \mathrm{TeV}$, https://cds.cern.ch/record/2758361.

[4] CMS collaboration, Search for top squarks in final states with two top quarks and several light-flavor jets in proton-proton collisions at $\sqrt{s}=13 \mathrm{TeV}$, Phys. Rev. D 104 (2021) 032006 [2102.06976].

[5] P. Baldi, K. Cranmer, T. Faucett, P. Sadowski and D. Whiteson, Parameterized neural networks for high-energy physics, Eur. Phys. J. C 76 (2016) 235 [1601.07913].

[6] Y. Ganin and V. Lempitsky, Unsupervised domain adaptation by backpropagation, 1409.7495. 